\title{
Effects of Kinesio Taping and Massage Application to Calf Muscle on Ankle Stability
}

\author{
Chan-Woo Kim, PT $\cdot$ Hyo-Lyun Roh ${ }^{1 \dagger}$ \\ Majored in Physical Therapy, Graduate School of Kangwon National University \\ ${ }^{1}$ Department of Physical Therapy, Kangwon National University
}

Received: July 12, 2018 / Revised: July 16, 2018 / Accepted: August 5, 2018

(C) 2018 J Korean Soc Phys Med

\section{| Abstract |}

PURPOSE: This study was conducted to investigate the effects of Kinesio taping and massage application to the calf muscles on ankle stability.

METHODS: The study subjects were 66 healthy adults (male: 32, female: 34) who had no instability in their ankles. Subjects were randomly assigned to a Kinesio taping group or a massage group. The research tool used the Y-balance test to measure instability of the ankle. The distance between the right and left foot was measured from the center in the anterior, posterior-medial, and posterior-lateral directions. Massage was applied to the calf muscles three times over two weeks and Kinesio tape was attached to the calf muscle and tibialis anterior for 10 hours twice during the experimental period.

RESULTS: The results showed that application of Kinesio taping resulted in increases in the distance between the feet increased in some directions. After the massage, the distance extended from the anterior and posterior-lateral directions was prolonged, and the ankle stability on the right and left

†Corresponding Author : Hyo-Lyun Roh bustryagain@naver.com, http://orcid.org/0000-0002-0187-7109 This is an Open Access article distributed under the terms of the Creative Commons Attribution Non-Commercial License (http://creativecommons.org/licenses/by-nc/3.0) which permits unrestricted non-commercial use, distribution, and reproduction in any medium, provided the original work is properly cited. sides was partially improved by massage application.

CONCLUSION: Kinesio taping and massage applied to the calf muscles are appropriate interventions for the improvement of ankle stability. Based on the application times of massage, Kinesio taping appears to work more effectively for ankle stability.

Key Words: Ankle stability, Calf muscle, Kinesio taping, Massage, Y-balance test

\section{Introduction}

The ankle joint palpates the sensory feedback for posture maintenance through synergic work of the lower limbs muscles and sensation of the weight load and soles (Menz et al., 2003). The ankle joint enables stepping forward while walking (Mansfield and Neumann, 2014). In normal adults, the ankle joint strategy and muscular coordination are primarily used for vertical control. To accomplish this, the gastronomic muscle is activated first in the case of anterior instability, while the tibialis anterior is activated for posterior instability (Horak, 1987). When the ankle strategy is difficult to maintain, the hip strategy is used, and the posture strategy is used in the order of footing (Shumway-Cook and Horak, 1986). However, despite this posture strategy, ankle joint sprains occur because of severely rugged floor surfaces, rapid shaking speed, and 
muscle problems around the ankle. Ankle instability, which is characterized by recurrent giving way, often develops after repeated lateral ankle sprains (Mervat et al., 2016). The best way to prevent chronic ankle instability is through initial treatment of ankle sprains.

Various methods have been suggested to prevent ankle injuries and solve the problem of instability. Among them, Kinesio taping and massage are easily applicable methods. Kinesio taping relieves pain and restores function and muscle strength by attaching taping to muscles. Previous studies have shown that ankle joint taping is the most effective method for reducing the incidence of repeated injury in subjects with a history of ankle joint sprain (Dizon and Reyes, 2010). This method is supposed to improve local circulation, reduce edema, facilitate or relax muscles, and improve joint function by enhancing sensory mechanisms (Slupik et al., 2007). Utilization of this method is high because it has immediate effects and low side effects (Roh, 2010). In addition, when tape is attached to the ankle joint it improves the sensory input about proprioceptive, even in the absence of weight-bearing; therefore, it can improve balance and walking ability by positioning the joint precisely (Seo et al., 2014).

Massage stimulates the function control of the reflexive nervous system and calms abnormal excitability through bare-hand stimulation. Therefore, it is commonly used to recover muscles and joints when there is excessive tension, spasms and pain in the muscles. Additionally, massage improves flexibility, strength and endurance of athletes to maximize their performance (Park et al., 2017). Massage can be performed throughout the human body, but it is commonly applied to large muscles. In the case of lower limbs, it is applied to large muscles such as the hamstring, hip adductor, hip abductor, quadriceps, tibialis anterior, calf muscle, tibialis posterior and peroneus (Eo and Shin, 1985).

The calf muscle is an important muscle that maintains the center point of mass of the basal plane through continuous muscle contraction while walking (Jo et al.,
2017). This muscle produces sufficient strength to maintain stability during exercise of the lower limb and has a great influence on dynamic balance (Youm and Kim, 2012). The soleus moves when conducting activities that require relatively low power over a long period of time, such as adjustments for standing or posture fluctuation, providing ankle stability (Mansfield and Neumann, 2014). Thus, massage of the calf muscles is regarded as a method for improving ankle stability.

This study was conducted to investigate the effects of Kinesio taping and massage on calf muscles to improve ankle stability. Through this, a method was proposed to increase ankle stability and prevent injuries by using massage and Kinesio taping therapy.

\section{Method}

\section{Study design}

This investigation was a single blind randomized control trial study of the effects of lower limbs Kinesio taping and calf massage on ankle stability.

\section{Subjects of study}

This study investigated 66 university students who fully listened to the purpose of the study and spontaneously agreed to participate actively. Thereafter, the study subjects had not experienced musculoskeletal diseases for the last 12 months, had no orthopedic problems in their lower limbs and had no neurological impairments or physical sensory abnormalities.

A total of 66 subjects (32 males and 34 females) were selected in accordance with the criteria and divided into two groups using random allocation software (version 1.0) (Saghaei, 2004). The general characteristics of the subjects are shown in Table 1.

\section{Study process}

Subjects were asked to wear simple t-shirts and shorts 
Table 1. General Characteristics of the Subjects

\begin{tabular}{cccc}
\hline Sex & Age $(\mathrm{yr})$ & Height $(\mathrm{cm})$ & Weight $(\mathrm{kg})$ \\
\hline Male $(\mathrm{n}=32)$ & $21.5 \pm 4.5$ & $173 \pm 7$ & $66 \pm 17$ \\
Female $(\mathrm{n}=34)$ & $21 \pm 30$ & $161.75 \pm 6.25$ & $51.75 \pm 8.25$ \\
Total $(\mathrm{n}=66)$ & 21.25 & 167 & 58.88 \\
\hline
\end{tabular}

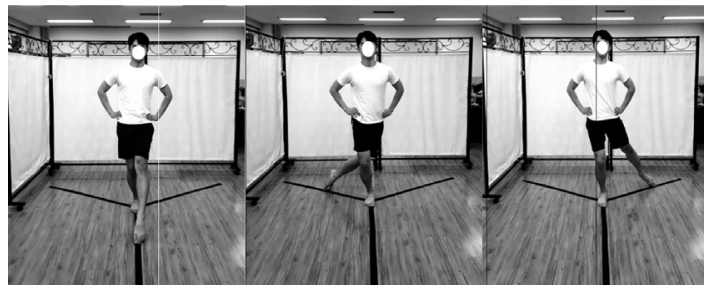

Fig. 1. Y Balance test

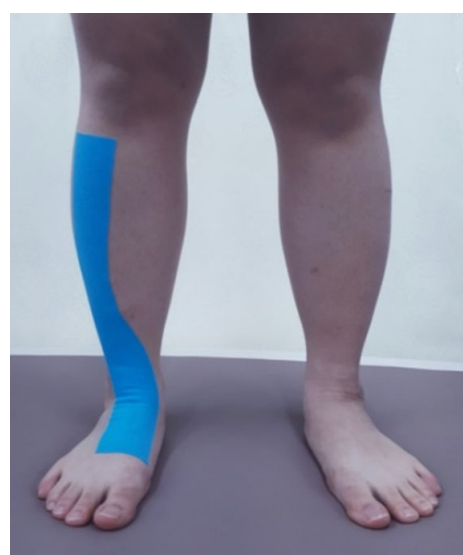

Fig. 2. Kinesio taping on the tibialis anterior

to avoid discomfort when applying massage and Kinesio taping and were instructed to refrain from intense activities during the study. Massage was applied three times for two weeks. All subjects applied methods such as police law, attention method, and stocking method according to the method of Park et al. (2017). Massage was applied to one calf for 3 minutes at a time for a total of 6 minutes while in the prone position by two female physiotherapists qualifications in massage.

Kinesio taping was conducted using $5 \times 30 \mathrm{~cm}$ tape attached to the calf muscle and tibialis anterior for 10 hours twice during the study period (Fig. 1, 2). Additionally,

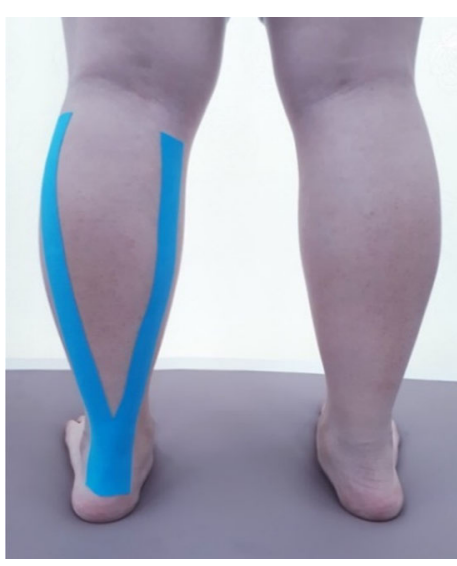

Fig. 3. Kinesio taping on the Calf muscle

a Y-shaped was attached to the other side of the calf muscle in line with the venter, resulting in a linear shape attached to the tibialis anterior. At this time, Kinesio tape was properly attached to the muscle area (Thuy and Clint, 2017).

The Y Balance Test (YBT) was measured before and after applying the massage and before and kinesio tape was applied and 10 minutes after attachment (Fig. 3).

\section{Instrument of evaluation}

A Y Balance test was performed to evaluate ankle joint stability before and after applying tape and massage. This test does not require a machine and can be conducted at low cost. The YBT, which has been utilized as a screening tool to predict injury because of aberrant neuromuscular control (Plisky et al., 2006), was developed to improve reliability and standardize performance of the modified SEBT (Plisky et al., 2009). The YBT assesses performance during single-leg balance while conducting reaching tasks in the anterior (ANT), posteromedial (PM), and posterolateral (PL) directions (Plisky et al., 2009) to determine lower 
extremity movement asymmetry and balance deficits (Gribble et al., 2012; Plisky et al., 2009).

Measurements were performed using the bottom line as shown in Fig. 2. Subjects were tested while barefoot. The supporting foot was positioned at the center point of the heel, while the big toes were in contact with the straight line. Subjects stood while their foot was adjusted to the central part where the line meets three times. When the experimenter's arm was positioned on the pelvis to maintain the posture (Herrington et al., 2009).

While the big toe of the outstretched foot touched the line lightly, test inspectors marked the touch point. The outstretched foot was then repositioned to the starting position. At this time, the subject should not lose balance. During return of the outstretched foot, the supporting foot should remain at the midpoint (Herrington et al., 2009). The balance ability will be great as much as stretching one's feet further. Measurements were performed three times in one direction using the mean value. After 3 seconds of rest, the foot was moved to the next direction. If the foot deviated from the center of the grid during the test or balance was lost, the measurement was performed again (Munro and Herrington, 2010).

\section{Data Analysis}

For this study, the statistical program SPSS 22.0 was used to analyze the data. The general characteristics of the subjects were calculated using descriptive statistics and the mean and standard deviation were examined.

The taping group and the massage group were compared using independent sample t-tests. In addition, corresponding sample t-tests to identify differences before and after intervention. Statistical significance was set at $\mathrm{p}<.05$.

\section{Results}

This study was conducted to investigate the effects of calf massage and Kinesio taping on ankle stability. The results are as follows.

1. Changes in ankle stability before and after applying Kinesio Taping

Before and after applying Kinesio Taping to the calf muscles, YBT was used to examine the ankle stability of the right and left ankle.

After applying Kinesio taping, there were significant differences $(p<.01)$ in the anterior $(p<.05)$, posterior-medial, and posterior-lateral directions. There were no significant differences in the anterior and posterior-medial directions, even when the right foot was supported. However, there were significant differences in the posterior-lateral direction $(\mathrm{p}<.01)$ (Table 2).

After applying Kinesio Taping, the distance between the center of the foot and the other foot in some directions has increased.

Table 2. Changes in Y-Balance after Taping

(n: 33 , unit: $\mathrm{cm}$ )

\begin{tabular}{|c|c|c|c|c|c|}
\hline \multirow{2}{*}{ Supported leg } & & \multicolumn{2}{|c|}{ Mean \pm SD } & \multirow{2}{*}{$\mathrm{t}$} & \multirow{2}{*}{$\mathrm{P}$} \\
\hline & & Pre & Post & & \\
\hline \multirow{3}{*}{$\mathrm{Lt}$} & Anterior & $68.16 \pm 6.21$ & $67.63 \pm 5.40$ & .605 & .55 \\
\hline & Posterio-medial & $71.71 \pm 9.42$ & $76.08 \pm 9.77$ & -3.44 & .00 \\
\hline & Posterio-lateral & $74.85 \pm 8.91$ & $79.22 \pm 8.65$ & -4.85 & .00 \\
\hline \multirow{3}{*}{ Rt } & Anterior & $65.68 \pm 5.12$ & $67.46 \pm 4.92$ & -2.20 & .04 \\
\hline & Posterio-medial & $72.11 \pm 8.88$ & $76.90 \pm 9.18$ & -5.98 & .00 \\
\hline & Posterio-lateral & $75.43 \pm 7.86$ & $77.76 \pm 8.37$ & -3.46 & .00 \\
\hline
\end{tabular}




\begin{tabular}{|c|c|c|c|c|c|}
\hline \multirow{2}{*}{ Supported leg } & & \multicolumn{2}{|c|}{ Mean \pm SD } & \multirow{2}{*}{$\mathrm{t}$} & \multirow{2}{*}{$\mathrm{p}$} \\
\hline & & Pre & Post & & \\
\hline \multirow{3}{*}{$\mathrm{Lt}$} & Anterior & $77.26 \pm 4.60$ & $81.79 \pm 4.93$ & -5.44 & .00 \\
\hline & Posterio-medial & $68.82 \pm 13.93$ & $77.28 \pm 15.36$ & -6.08 & .00 \\
\hline & Posterio-lateral & $72.51 \pm 14.42$ & $78.75 \pm 14.83$ & -5.61 & .00 \\
\hline \multirow{3}{*}{ Rt } & Anterior & $79.60 \pm 5.26$ & $83.28 \pm 4.87$ & -5.58 & .00 \\
\hline & Posterio-medial & $75.06 \pm 12.71$ & $78.06 \pm 17.46$ & -1.16 & .25 \\
\hline & Posterio-lateral & $70.040 \pm 14.60$ & $77.89 \pm 16.68$ & -5.27 & .00 \\
\hline
\end{tabular}

2. Changes in ankle stability before and after massage application

Before and after massaging calf muscles, the stability of the ankles on the right and left sides was examined using YBT (Table 3).

There were significant differences between the anterior, posterior-medial, and posterior-lateral directions in the state of supporting the left foot after application of massage $(p<.05)$. However, there was no significant difference in the posterior-inferior aspect or posterior-lateral aspect of the right foot $(\mathrm{p}<.01)$.

After the massage, the distance extended from the anterior and posterior-lateral directions was prolonged, and the ankle stability on the right and left sides was partially improved by massage application.

\section{Comparison of Ankle Stability of the Massage Group and Kinesio Taping Group}

We used an independent t-test based on difference values before and after the intervention to determine the differences between the massage group and the kinesio taping group.

There was no significant difference between the two groups in the anterior direction when supporting the left side $(\mathrm{p}<.01)$ and in the posterior-medial and posteriorlateral directions. There was also no significant difference in the anterior and posterior-medial direction when supporting the right side $(\mathrm{p}<.05)$. Finally, there was no significant difference between the two groups.

Overall, the massage group showed slightly more improvement in ankle stability than the Kinesio taping group.

\section{Discussion}

This study was conducted to examine the effects of Kinesio taping and topical massage of the calf muscle on ankle stability in normal adults. After application of Kinesio taping, the distance from the left and right ankles was increased in all directions except the posterior-lateral direction. As a result, Kinesio taping seems to have improved the stability of the right and left ankles. These results are similar to those reported by Elgen and Ulkar (2008), who suggest that taping has a positive effect on posture control by limiting excessive movement of the joints and providing somatosensory feedback. In addition, to increasing the stability of the ankle joint, taping led to an improvement in posture center, which was similar to the results of a study conducted by Jo et al. (2017). Mervat et al. (2016) also reported that Kinesio tape had positive effects on patients with first degree ankle sprain and could be used safely to improve ankle joint stability. Overall, Kinesio taping improved posture control function.

The application of Kinesio tape increases the postural balance index, shortens the muscle mobilization time and improves the inherent receptive sensory feedback 
Table 4. Comparison between Massage and Taping in Changes of Y-balance Test

(unit : $\mathrm{cm}$ )

\begin{tabular}{|c|c|c|c|c|c|}
\hline \multirow{2}{*}{ Supported leg } & & Massage group & Taping group & \multirow{2}{*}{$\mathrm{t}$} & \multirow{2}{*}{$\mathrm{P}$} \\
\hline & \multicolumn{3}{|c|}{ Mean \pm SD } & & \\
\hline \multirow{3}{*}{$\mathrm{Lt}$} & Anterior & $-4.53 \pm 5.00$ & $-.06 \pm 5.74$ & -3.43 & .00 \\
\hline & Posterio-medial & $-8.461 \pm 8.35$ & $-4.90 \pm 7.79$ & -1.81 & .07 \\
\hline & Posterio-lateral & $-6.24 \pm 6.67$ & $-4.62 \pm 5.24$ & -1.10 & .28 \\
\hline \multirow{3}{*}{ Rt } & Anterior & $-3.68 \pm 3.96$ & $-1.93 \pm 4.80$ & -1.65 & .10 \\
\hline & Posterio-medial & $-3.00 \pm 15.53$ & $-4.87 \pm 4.69$ & .66 & .51 \\
\hline & Posterio-lateral & $-7.94 \pm 9.04$ & $-3.40 \pm 4.55$ & -2.66 & .01 \\
\hline
\end{tabular}

mechanism, thereby increasing proprioceptive sense (Hinman et al., 2003). However, Travis et al. (2004) reported that the application of Kinesio tape did not enhance proprioception in healthy individuals at the ankle in the motions of plantar flexion and $20^{\circ}$ of plantar flexion with inversion. The taping is controlled by muscle contraction caused by the resting motor reflex (Choi et al., 2007) and continuous stimulation to the surrounding muscles and joints provides feedback for information regarding muscular contraction. Therefore the tape maintains joint stability and helps improve balance.

In this study, the extended range of the foot in the anterior and posterior-lateral directions after applying massage appeared to improve stability of the right and left ankle. Although the effects of massage on the ankle flexion and flexibility of the ankle joint did not change significantly after massage treatment in the present study, Yook (2009) reported that there was some increase in the flexibility of the ankle joint. Moreover, Park et al. (2017) reported that the balance and ankle flexibility increased after calf massage, which is in accordance with the results of the present study.

Massage improves venous blood flow and facilitates the flow of lymphatic fluid (Caffarelli et al., 1985), while also reducing edema, altering cell membrane permeability and enhancing the metabolic function of the muscles through smooth control of blood circulation (Park and Ahn, 2010). Therefore, muscle fatigue recovery and muscle activity can be conducted smoothly and massage application is sufficient to cause a physiological response to local tissues and mechanical massage application increases intramuscular blood flow. In addition, sports massage increases the size of the muscles and causes the them become harder and more elastic (Kim et al., 2013).

In this study, ankle stability improvement was higher in the massage group than in the Kinesio taping group in the anterior direction when supporting the left side and in the posterior direction when supporting the right side. However, taping was compared before and after single application in this study, while massage was performed three times over 2 weeks. Considering the duration and frequency of intervention, it is difficult to say that the massage treatment was effective. If the massage is applied only one time, it is likely that taping would be more effective. Indeed, the Kinesio taping group was evaluated at 10 minutes after applying the tape and there seemed to be an immediate effect. In addition, since taping affects the mechanical receptors of the continuous muscles and joints during application (Hopper et al., 2009), it will be more effective even though the application method is simpler.

It should be noted that this study was limited in that it was difficult to accurately quantify the effects of the two methods because taping was applied once and massage was applied several times. Moreover, a value based on measurement of the subject's leg length is often used; 
however, this study did not measure leg length.

\section{Conclusion}

This study was conducted to investigate the effects of Kinesio taping and massage application on ankle stability. Both treatments improved ankle stability; therefore, massage and taping of the lower limbs will help stabilize the ankle joint and restore balance.

\section{Acknowledgements}

This study was supported by 2017 Research Grant from Kangwon National University (No. 620170133).

\section{References}

Caffarelli L, Nirenberg L, Spruck J. The Dirichlet problem for nonlinear second order elliptic equations, III: Functions of the eigenvalues of the Hessian. Acta Math. 1985;155(1):261-301.

Choi PB, Lee BS, Kim BJ, et al. Effect of Kinesio-taping after muscle fatigue on proprioception, blood lactic acid and muscle soreness. J coach develop. 2007; 9(3):209-17.

Dizon J, Reyes J. A systematic review on the effectiveness of external ankle supports in the prevention of inversion ankle sprains among elite and recreational players. J Sci Med Sport. 2010;13(3):309-17.

Elgen E, Ulkar B. Proprioception and ankle injuries in soccer. Clini Spor Medi. 2008;27(1):195-217.

Eo KH, Shin KM. Therapeutic massage for athlete. Kore J Spor medi. 1985;3(1):31-41.

Gribble PA, Hertel J, Plisky P. Using the Star Excursion Balance Test to assess dynamic postural-control deficits and outcomes in lower extremity injury: a literature and systematic review. J Athl Train. 2012; 47(3): 339-57.

Horak FB. Clinical measurement of postural control in adults.
Phys Ther. 1987;67(12):1881-5.

Herrington L, Hatcher J, Hatcher A, et al. A comparison of Star Excursion Balance Test reach distances between ACL deficient patients and asymptomatic controls. Knee. 2009;16(2):149-52.

Hinman RS, Crossley KM, McConnell J, et al. Efficacy of knee tape in the management of osteoarthritis of the knee: blinded randomised controlled trial. Briti Medi Jour. 2003;327(7407):135.

Hopper D, Samsson K, Hulenik T, et al. The influence of Mulligan ankle taping during balance performance in subjects with unilateral chronic ankle instability. Phys Ther Spor. 2009;10(4):125-30.

Jo TS, Kim TH, Choi HS, et al. The effect of elastic therapentic taping on lower limb kinematics during a cross cutting movement from landing in subjects with chronic ankle instability. J Korean Soc Phys Med. 2017;12(4):1-9.

Kim GC, Lee JH, Kwon SM. Effects of hamstring flexibility and dynamic stability of lower lumbar according to stretching and massage techniques. J Korean Soc Phys Med. 2013;8(4):609-17.

Mansfield PJ, Neumann DA. Essentials of Kinesiology for the Physical Therapist Assistant, (2nd ed). USA. Elsevier Inc. 2014.

Menz HB, Lord SR, Fitzpatrick RC. Acceleration patterns of the head and pelvis when walking on level and irregular surfaces. Gait Posture. 2003;18(1):35-46.

Mervat AM, Nadia LR, Al Shimaa RA. Effect of Kinesio-taping on ankle joint stability. Interna J Med Res \& Health Sci. 2016;5(5):51-8.

Munro AG, Herrington LC. Between-session reliability of the star excursion balance test. Physical Therapy in Sport. 2010;11(4):128-32.

Park JU, Shim JM, Kim SJ, et al. Application of massage for ankle joint flexibility and balance. J Physi Thera Scie. 2017;29(5):789-92.

Park IB, Ahn SY. The effects of exercise with taping on muscle strength and bone mineral density, IGF-1 in blood 
of elderly women. J Korean Soc Phys Med. 2010; 5(2):289-300.

Plisky PJ, Gorman PP, Butler RJ, et al. The reliability of an instrumented device for measuring components of the star excursion balance test. N Am J Sports Phys Ther. 2009;4(2):92-9.

Plisky PJ, Rauh MJ, Kaminski TW, et al. Star Excursion Balance Test as a predictor of lower extremity injury in high school basketball players. J Orthop Sports Phys Ther. 2006;36(12):911-9.

Roh HL. Effects of taping therapy and passive range of motion exercises on shoulder joint, hand dexterity in the elderly. J Kore Acade Indus Coopera Socie. 2010; 11(7):2468-74.

Saghaei M. Random allocation software for parallel group randomized trials. BMC Medi Rese Metho. 2004; 4(26):1-6.

Seo HJ, Kim JH, Son KK, et al. The effect of gluteal taping on posture and balance during standing in children with hemiplegic cerebral palsy. J Korean Soc Phys Med. 2014;9(4):391-8.
Slupik A, Dwornik M, Bialoszewski D, et al. Effect of Kinesio Taping on bioelectrical activity of vastus medialis muscle. Preliminary report. Ortop Traumatol Rehabil. 2007;9(6):644-51.

Shumway-Cook A, Horak FB. Assessing the influence of sensory interaction on balance. Phys Ther. 1986; 66(10):1548-50.

Thuy B, Clint B. Length, Strength and Kinesio Tape : Muscle Testing and Taping Interventions. Australia. Elsevier. 2017.

Travis H, John WM, Mark D, et al. The effects of Kinesio TM Taping on proprioception at the ankle. J Spo Sci Med. 2004;3(1):1-7.

Yook CY. Effect of massage on the flexibility of ankle joint and spastic plantar flexor. J Kore Spor Resea. 2009;20(3):69-76.

Youm CH, Kim TH. Effects of induced fatigue of ankle joint muscle on the capability and recovery of postural control during single-leg stance. Kore J Spor Biomech. 2012;22(2):219-28. 